\title{
REAL WORLD DATA ON PATIENT PROFILES AND PATTERNS OF CARE IN DE NOVO METASTATIC BREAST CANCER: AN ANALYSIS OF 17 YEARS (1995-2011)
}

Leonardo R. Soares', Ruffo Freitas-Junior¹, Rodrigo D. Nunes¹, Edesio Martins', José C. Oliveira1, Maria P. Curado 'Population-Based Cancer Registry of Goiânia, Goiás Association to Cancer Combat - Goiânia (GO), Brazil.

Objectives: To describe the epidemiological profile of women with de novo metastatic breast cancer (MBC) in Goiânia, using the incidence data from the population-based cancer registry (PBCR). Methodology: An ecological study conducted at the PBCR in Goiânia, Brazil, from 1995 to 2011. We included women with MBC at diagnosis and calculate the standardized incidence rate and the annual percentage change (APC) in the period. Clinical, demographic, diagnostic and treatment characteristics were analyzed in two periods (1995-2003 versus 2004-2011). Results: There were 5,289 cases of breast cancer, of these 277 (5.2\%) in the metastatic stage. The adjusted incidence was 8.9/100,000 in 1995 and 6.04/100,000 in 2011 (APC -1.1, p=0.6). There was no difference in the incidence of metastatic breast cancer in the two periods studied. The majority of the patients were assisted by the public health system $(70.3 \%)$ and the mean age at diagnosis was $54.7( \pm 14.5)$ years. It was possible to analyze a subpopulation of 156 patients in the two main treatment centers in the city. By the immunohistochemistry, 52 (70.2\%) women had hormone receptor positive (HR+), of which 14.0\% (6/43) received endocrine therapy in the first line of systemic treatment and 48.5\% (17/35) in second-line. Ten patients underwent resection of metastatic lesion (10/108, 9.2\%). Comparison of clinical data between the periods 1995-2003 and 2004-2011 revealed no significant differences in relation to age, histological grade, staging, presence of symptoms at diagnosis, or treatment. Conclusion: Our study included a MBC population with predominantly locally advanced tumors and Luminal-like phenotype. The incidence rate of MBC in Goiânia did not change over the 17-year period. Most of the cases received chemotherapy in the first line of systemic treatment, independent of the tumor phenotype. 